DOI: $10.35643 /$ Info.24.2.4

Artículo Original

\title{
Impacto del Plan Ibirapitá sobre la brecha gris en Uruguay.
}

\section{Impact of the Ibirapitá Plan on the Gray Gap in Uruguay.}

\section{Soledad Caballero ${ }^{1}$}

${ }^{1}$ Licenciada en Comunicación Social (UCU). Ayudante grado 2 del Departamento de Teoría y Metodología del Instituto de Comunicación de la Facultad de Información y Comunicación de la Universidad de la República (FIC-Udelar) y Asistente grado 1 del Instituto de Información de la FIC. Correo electrónico: soledad.caballero@fic.edu.uy. ORCID: 0000-0002-9301-5329

\section{Resumen}

Se predice el impacto que tiene el Plan Ibirapitá —en tanto política pública orientada a superar la divisoria digital- sobre la brecha gris que se constata entre las mujeres de 65 años o más en Uruguay. De acuerdo a este modelo, la divisoria digital representa la principal barrera para el uso de Internet entre las mujeres de 65 años o más beneficiarias del Plan Ibirapitá. El mayor impacto individual entre las variables de la infraestructura tecnológica consideradas es el de la tableta Ibirapitá. Sin embargo, las predicciones del modelo indican que su incidencia sobre la probabilidad de uso de Internet está sujeta a la disponibilidad de conexión a Internet en el hogar.

A su vez se confirmó la incidencia de la estructura del hogar y de la red familiar sobre la probabilidad de uso de Internet. La motivación de tener un hijo lejos, en el exterior, incide positivamente sobre la probabilidad de uso de Internet, al igual que la presencia de pareja en el hogar y la vida en un hogar unipersonal. No obstante, la presencia de hijos o de menores de 14 años en el hogar disminuye la probabilidad de uso de Internet. De hecho, a pesar de tener computadora e Internet en el hogar, cuando las mujeres de perfil promedio analizado conviven con menores de 14 años o con hijos en el hogar, no usan Internet. En dicho escenario sin divisoria digital, la brecha gris es superada con la tableta del Plan Ibirapitá.

Palabras claves: Plan Ibirapitá; Brecha gris; Divisoria digital; Personas mayores; Uruguay.

\begin{abstract}
We predict the impact of Ibirapita Plan as a public policy oriented towards overcoming the grey digital divide in Uruguay. According to this logistic model, the digital divide is the most critical barrier for the use of Internet among women 65 years and above targeted by this public policy. The Ibirapita tablet has the major impact among the technical infrastructure variables considered in the model. However, its impact depends on the availability of Internet access at home. Besides, we proved the household structure and family composition impact over the probability of use of the Internet among this female population. On the one
\end{abstract}


side, the motivation of a son living abroad has a positive influence, as well as a partner living at home or living alone. On the other side, living with their own children at home and with children below 14 years old diminishes the probability of Internet use. As a matter of fact, even women who have a computer and Internet access at home do not use Internet when they live with minors or their offspring. In such case, the grey digital divide is defeated by Ibirapita Plan.

Key words: Ibirapita Plan; Grey digital divide; Elderly people; Digital divide; Uruguay.

Fecha de recibido: 20/02/2019

Fecha de aceptado: 28/03/2019

\section{Introducción}

La divisoria digital remite a las inequidades en el acceso y el uso de las tecnologías digitales (Scheerder, van Deursen y van Dijk, 2017) y la expresión «brecha gris» a la poca cantidad de personas mayores que usa Internet a pesar de la alta penetración de esta tecnología digital (Friemel, 2016; Millward, 2003). Existen varios factores que mantienen dicha brecha (Blaschke, Freddolino y Mullen, 2009), de los cuales los demográficos y los socioeconómicos han sido los más estudiados, pero es necesario prestar mayor atención a las condiciones de vida de los mayores tanto en las investigaciones como en las políticas de inclusión (De Benito-Castanedo, 2017; van Deursen, Helsper y van Dijk, 2017; Scheerder et al., 2017).

Actualmente, en los países desarrollados, la principal causa de la brecha gris es la falta de habilidades de los mayores para usar Internet, a pesar de que tienen más oportunidades de aprendizaje que en los países en desarrollo. En estos, la enseñanza de Internet dirigida a mayores tiene resultados diversos según el contexto; la mejora de la infraestructura, el uso de dispositivos móviles y los bajos costos de acceso muestran un impacto positivo. En general, las razones para explicar la falta de habilidad en el uso de Internet son las mismas que en los países desarrollados, aunque se agregan barreras culturales, relacionadas con bajos niveles educativos y la falta de infraestructura (Mubarak y Nycyk, 2017).

Si bien los costos y la disponibilidad de acceso siguen siendo las barreras críticas para el aumento de la penetración de Internet, hay una combinación de factores demográficos y de capital humano que frenan el crecimiento de Internet en Colombia, Ecuador, México y Perú (Galperin, 2017). En el caso de las personas mayores, la mera tenencia del dispositivo de acceso y la superación de la divisoria digital no es condición suficiente para que exista inclusión digital. Existen otros factores que inciden en el mantenimiento de esta barrera. Frecuentemente, los mayores no usan Internet por falta de interés, porque consideran que no les va a ser útil o incluso porque dudan de su propia capacidad para aprender a usar la tecnología (Helsper, 2009; Millward, 2003). Aunque el aprendizaje de tecnologías en la vejez es posible, los cambios en el funcionamiento cognitivo (enlentecimiento en el procesamiento de información, declive en algunos aspectos de la memoria y en las funciones ejecutivas), sumados a ciertas limitaciones funcionales (déficit visual, barreras ergonómicas), pueden presentarse como 
obstáculos para el uso de Internet (Petretto et al., 2016; Choi y DiNitto, 2013; Binotti y Spina, 2009).

El aprendizaje de tecnologías entre los adultos mayores representa un gran desafío, dado que, en general, tienen menor memoria, menor desempeño y necesitan más tiempo para adquirir cosas nuevas (Czaja y Sharit, 2012; Haederle, 2011; World Health Organisation, 2002). Por este motivo, la repetición es particularmente importante en la enseñanza a personas mayores (Chaffin y Harlow, 2005). De hecho, para que se produzca el aprendizaje entre los mayores es fundamental la actividad de las funciones cerebrales ejecutivas, la observación crítica del proceso, junto con la evaluación, la corrección de errores y la modificación de acciones y comportamientos necesarios para resolver situaciones nuevas (Petretto et al., 2016; Binotti y Spina, 2009).

Mientras al envejecer la velocidad de aprendizaje y la memoria decrecen, lograr usar tecnologías implica incorporar múltiples habilidades al mismo tiempo (Merriam, Caffarella y Baumgartner, 2012). A pesar de estos desafíos que enfrentan para aprender a usar una nueva tecnología, no existe un menor deseo de instruirse entre las personas mayores (Broady, Chan y Caputi, 2010), quienes tienen la capacidad de seguir formándose gracias, en gran parte, al proceso de plasticidad cerebral (Aldana, García y Jacobo, 2016).

Los adultos mayores precisan motivaciones para aprender a usar tecnologías digitales y necesitan encontrar un vínculo claro con las aplicaciones prácticas que le puedan dar a estas tecnologías en su vida cotidiana (Merriam et al., 2012; Chaffin y Harlow, 2005). Los factores de aceptación de tecnologías de la información que se destacan en los estudios referidos a adultos mayores son el beneficio percibido, la influencia de la familia y la influencia social, el control de conducta percibido y la usabilidad percibida, además de los mediadores sociodemográficos, como género, edad, educación, ingresos, estado civil, salud y experiencia (Ma et al., 2015). La teoría STAM (Senior Technology Acceptance \& Adoption Model) de Renaud y Van Biljon (2008) señala como uno de los factores que facilitan el aprendizaje y el uso de tecnologías entre las personas mayores al soporte social disponible que les ayude a aceptar la tecnología (Tsai, Shillair y Cotten, 2015; Barnard et al., 2013). El soporte social refiere al intercambio de diferentes tipos de recursos a través de las relaciones sociales (Phillips, Ajrouch y Hillcoat-Nallétamby, 2010) y, entre los adultos mayores en Uruguay, esto remite a la familia. Además, las tecnologías en sí mismas se consideran un tipo de recurso (Silva, 2010). El soporte social interesa no solo por su rol en la adopción de tecnologías entre los mayores (Damodaran y Sandhu, 2016; Tsai et al., 2015), sino también porque es amplia la evidencia de su vinculación con distintos aspectos del bienestar de las personas (por ejemplo, la salud). También para la teoría cognitiva social el apoyo del entorno, entendido como la familia, los amigos, las personas importantes para la persona mayor, resulta clave en el proceso de aprendizaje (Bandura, 1977, 1994).

\section{Antecedentes}

El uso de Internet entre adultos mayores se encuentra estratificado por sexo, edad, nivel educativo, ingresos y estado civil (Hargittai y Dobransky, 2017; Chang, 
McAllister y McCaslin, 2015). Además de los mediadores sociodemográficos, entre los factores de aceptación de tecnologías, Ma et al. (2015) destacan la usabilidad, el control y el beneficio percibido, la influencia social y de la familia. Los últimos aluden al soporte social disponible, es decir, al intercambio de diferentes tipos de recursos a través de relaciones sociales (Phillips et al., 2010). Este apoyo es un factor facilitador del aprendizaje y del uso de tecnologías entre personas mayores (Damoradan y Sandhu, 2016; Tsai, et al., 2015; Barnard et al., 2013). Los hijos se destacan como estímulo y fuente de enseñanza en el uso de Internet, especialmente a mayor edad y menor nivel socioeconómico de los padres (Chang et al., 2015; Correa et al., 2013). Las mujeres mayores, en concreto, reciben más ayuda de sus hijos para usar Internet que los hombres (Correa et al., 2013). Además, el ciclo de vida familiar incide en la adopción de tecnologías en el hogar, que es el principal lugar de acceso a Internet entre los mayores (Mori y Harada, 2010; Instituto Nacional de Estadística [INE], 2017). Por ejemplo, la adopción de una computadora se asocia con características claves del ciclo de vida del hogar, como la presencia de niños en la casa o la vida en pareja (Brown, Venkatesh y Bala, 2006).

En Uruguay, las mujeres de 65 años o más que tienen hijos viviendo en el exterior, así como quienes conviven con una pareja en el hogar y quienes viven solas, tienen más chance de usar Internet que las que no tienen hijos en el exterior ni pareja en el hogar, respectivamente; mientras las que tienen hijos en el hogar tienen menos probabilidad de usar Internet (Caballero, 2014, 2016 y 2017). En Buenos Aires, Lima y Ciudad de Guatemala también se relacionó la estructura del hogar con el acceso a Internet de los mayores, ${ }^{1}$ en un estudio que indica que «la presencia de menores en el hogar incide en la decisión inicial de usar Internet, mientras que vivir con la pareja tiene un efecto la intensidad de uso de Internet» (Barrantes y Cozzubo, 2017, p. 14). Si bien los resultados de dicho trabajo son positivos en relación con la presencia de menores en el hogar, los autores refieren a varios estudios que dan cuenta de la ambigüedad que representan las relaciones intergeneracionales para la adopción y el uso de tecnologías digitales entre los adultos mayores (Barrantes y Cozzubo, 2017).

Para usar tecnologías, los mayores tienen necesidades básicas (acceso, soporte) y no instrumentales, que son más persistentes (contacto con las personas que importan, disfrutar del tiempo libre, avanzar en sus habilidades); ambas deben ser atendidas en las políticas públicas de inclusión (Ferreira, Sayago y Blat, 2016). Uruguay tiene una población envejecida (14\% tiene 65 años o más), $11 \%$ de jubilados y una amplia brecha gris: solo $7 \%$ de los usuarios de Internet tiene 65 años o más, porcentaje que se mantiene bajo (8\%) incluso cuando hay computadora e Internet en el hogar (consulta propia a Encuesta Continua de Hogares [ECH] del INE, 2017). Uruguay impulsa, desde 2015, una política pública de inclusión digital dirigida a jubilados que perciben un monto menor de mil dólares mensuales, denominada Plan Ibirapitá. Los beneficiarios reciben una tableta y un curso de tres horas, en forma gratuita.

En la población de mujeres beneficiarias del Plan Ibirapitá con 65 años o más, $52 \%$ tienen acceso a Internet en el hogar; $34 \%$ tienen computadora en el hogar

\footnotetext{
${ }^{1}$ Ambos estudios se realizaron con datos secundarios de los institutos de Estadística de los respectivos países.
} 
(no incluye tableta Ibirapitá); $58 \%$ tienen celular (smartphone o no), y $18 \%$ viven en un hogar donde hay una tableta Ibirapitá (de la cual puede ser beneficiario otro integrante del hogar). La edad promedio es 76 años y el ingreso per cápita promedio es 21390 pesos uruguayos. No tienen estudios formales o no terminaron la escuela primaria $28 \%, 54 \%$ tienen primaria completa y $18 \%$ terminaron el liceo o culminaron estudios superiores. $6 \%$ viven en zona rural, $30 \%$ viven solas, mientras que $38 \%$ tienen pareja en el hogar. $45 \%$ son viudas, $6 \%$ son solteras y $15 \%$ son divorciadas. $8 \%$ viven con menores de 14 años, $33 \%$ tienen hijos en el hogar y $13 \%$ tienen hijos en el exterior. De esta población, 27 \% usa Internet (consulta propia a ECH, INE, 2016, véase Tabla 1).

\section{Metodología}

Para definir qué se entiende por personas mayores, el uso de un criterio cronológico, como tener 65 años o más, resulta insuficiente, dado que no hay homogeneidad dentro de dicho grupo (Dannefer y Settersten, 2010; Dannefer, 1987). Abordar un estudio de personas mayores definiéndolas solo por la edad implica no dar lugar a la relevancia de aspectos vinculados al envejecimiento que han sido ampliamente estudiados por la comunidad científica. Con dicho criterio numérico se ensamblan grupos que envejecen de manera diferente.

Teniendo en cuenta la perspectiva del curso de la vida, las relaciones que se establecen desde los roles de género que se asumen a lo largo de la vida, junto con las experiencias acumuladas desde estos roles, nos modifican de manera tal que no es lo mismo llegar a la vejez siendo mujer o siendo hombre (Dannefer y Settersten, 2010; Helsper, 2010). Por ejemplo, hombres y mujeres disponen de diferente soporte social en la vejez, ayuda que la literatura indica que es relevante para el estudio del uso de Internet entre las personas mayores (Antonucci, Ajrouch y Birditt, 2014; Arber, Davidson y Ginn, 2003). Entonces, para considerar en alguna medida la heterogeneidad que hay en el criterio cronológico, se tuvo en cuenta el conocimiento científico vinculado al soporte social y a la teoría del curso de la vida, que indican al género como una variable relevante cuando se estudian personas mayores. Además, se consideraron las características demográficas del Uruguay, donde el envejecimiento es predominantemente femenino (INE, 2016). Con estos fundamentos, este análisis exploratorio se enfocó en las mujeres de 65 años o más beneficiarias del plan Ibirapitá. ${ }^{2}$

\section{Objetivos}

1. Identificar las variables que inciden sobre el uso de Internet entre las mujeres de 65 años o más beneficiarias del Plan Ibirapitá en Uruguay.

2. Analizar el impacto de la entrega de la tableta Ibirapitá sobre el uso de Internet entre las beneficiarias con perfil promedio.

\footnotetext{
${ }^{2}$ Este estudio se enmarca en el trabajo de doctorado enfocado en el uso de Internet entre mujeres de 65 años o más en Uruguay. Un avance de este modelo fue presentado en las II Jornadas de Investigación de la FIC (diciembre, 2017).
} 
3. Analizar si la entrega de la tableta Ibirapitá entre las mujeres de 65 años o más, con perfil promedio, es necesaria para alentar el uso de Internet cuando tienen Internet y computadora en el hogar.

4. Estudiar el impacto de la estructura del hogar sobre el uso de Internet.

Para responder a la primera pregunta de investigación se ajustó un modelo de regresión logística. La regresión logística permite «explicar o pronosticar la pertenencia [...] a los grupos definidos por la variable dependiente [...] en función de una o más variables independientes» (López-Roldán y Fachelli, 2015, p. 5). En este caso la variable dependiente es el uso de Internet y los grupos que define dicha variable son dos: a) usuaria de Internet, entendida como quien utilizó Internet en el último mes, y $b$ ) no usuaria de Internet. Las variables independientes, o predictores del uso de Internet, incluidas en el modelo logístico, están presentadas en la tabla 1 y contemplan aspectos relacionados con la infraestructura de acceso a Internet, aspectos demográficos, socioeconómicos, de la red familiar y estructura del hogar.

Se trabajó con datos secundarios de la ECH, INE (2016), la cual se lleva a cabo entre la población residente en hogares particulares de Uruguay, excluyendo residencias u otros hogares colectivos. Se utilizó el software SPSS 17 y el software RStudio versión 0.99.902. Se depuró la base filtrando los casos de beneficiarias de 65 años o más ${ }^{3}(n=5662)$. Se corrió un test GVIF y se verificó que no existieran problemas de multicolinealidad (véase Tabla 1). El máximo valor constatado fue 2,46, menor que el valor límite aceptado (Hair et al., 2010).

Tabla 1: Distribución de las variables del modelo logístico en la población estudiada y test de multicolinealidad

$\mathrm{n}=5662 \mathrm{~N}=139679$

Variable dependiente - Uso de Internet - $27 \%$

\begin{tabular}{r|l|c}
\hline \multicolumn{2}{c|}{ Variables independientes } & $\begin{array}{c}\text { Test } \\
\text { GVIF }\end{array}$ \\
\hline Edad (en años) & $\begin{array}{l}\text { Mínimo = 65 } \\
\text { Máximo = 98 } \\
\text { Promedio = 76 } \\
\text { Desviación estándar = 7,65 }\end{array}$ & 1.21 \\
\hline Nivel educativo & $\begin{array}{l}\text { 1- S/estudios o primaria incompleta - 27 \% } \\
\text { 2- Primaria completa - 54 \% } \\
\text { 3- Liceo completo o estudios superiores - 18 \% }\end{array}$ & 1,16 \\
\hline $\begin{aligned} \text { Ingresos per cápita } \\
\text { (en pesos uruguayos) }\end{aligned}$ & $\begin{array}{l}\text { Mínimo = 3748 } \\
\text { Máximo = 475 543 } \\
\text { Promedio = 21 390 }\end{array}$ & 1,27 \\
\hline
\end{tabular}

\footnotetext{
${ }^{3}$ Se seleccionó como beneficiarios del Plan Ibirapitá a jubiladas del BPS, bancarios, profesionales universitarios, Caja Notarial, Militar y Policial que percibían hasta 24416 pesos uruguayos (filtro propio a la ECH, INE, 2016).

${ }^{4}$ Se trabajó con la variable «ingresos per cápita» en base logarítmica, construida a partir de la variable «ingresos del hogar», considerando la cantidad de personas y la presencia de menores en el hogar. Se valoró la presencia de un menor como 0,5.
} 


\begin{tabular}{r|l|c}
\hline & Desviación estándar =13 395 & \\
\hline Hogar unipersonal & $30 \%$ & 2,46 \\
\hline Pareja en el hogar & $38 \%$ & 2,03 \\
\hline Hijos en el hogar & $33 \%$ & 1,71 \\
\hline Menores en el hogar & $8 \%$ & 1,18 \\
\hline Zona urbana o rural & $6 \%$ & 1,02 \\
\hline Hijos en el exterior & $13 \%$ & 1,02 \\
\hline $\begin{array}{r}\text { Tenencia de conexión } \\
\text { a Internet }\end{array}$ & $52 \%$ & 1,26 \\
\hline $\begin{array}{r}\text { Tenencia de } \\
\text { computadora }\end{array}$ & $34 \%$ & 2,36 \\
\hline Tenencia de celular & $58 \%$ & 1,09 \\
\hline $\begin{array}{r}\text { Tenencia de tableta } \\
\text { Ibirapitá }\end{array}$ & $18 \%$ & 2,05 \\
\hline
\end{tabular}

Todas las variables son dicotómicas, dummies (se presenta la frecuencia del valor 1), excepto: edad (cuantitativa), ingresos per cápita (cuantitativa) y nivel educativo (ordinal). Fuente: consulta propia a ECH, INE, 2016.

Para responder a las preguntas de investigación 2, 3 y 4 se corrió el modelo con un perfil promedio - siguiendo a Juan Ariel Bogliaccini y Federico Rodríguez (2015) - en distintos escenarios. En el perfil construido de beneficiaria del Plan Ibirapitá de 65 años o más se dejaron constantes en su valor promedio o mediana: la edad, el nivel educativo, de ingresos, la zona urbana y la tenencia de teléfono celular. Al dejar constantes los valores de estas variables, se pone foco en el estudio de la incidencia de las variables vinculadas a la infraestructura de acceso a Internet en el hogar, la tableta Ibirapitá, la estructura del hogar y la composición familiar en los cuatro escenarios propuestos:

a) hogares sin tableta Ibirapitá y sin infraestructura de acceso a Internet;

b) hogares con tableta Ibirapitá y sin infraestructura de acceso a Internet;

c) hogares sin tableta Ibirapitá y con infraestructura de acceso a Internet y

d) hogares con tableta Ibirapitá y con infraestructura de acceso a Internet.

Las variables de la infraestructura de acceso a Internet del hogar que se incorporan al análisis de los escenarios son la tenencia de conexión a Internet y de computadora en el hogar (que no es del Plan Ibirapitá). Los escenarios $a$ y $b$, en que los hogares donde viven las mujeres mayores no tienen computadora ni conexión a Internet, se denominan escenarios con divisoria digital; los escenarios $c$ y $d$, en que los hogares tienen conexión a Internet y computadora, se denominan escenarios sin divisoria digital.

Se utilizó el paquete Zelig del software R para aplicar el modelo logístico, simular estos escenarios con y sin divisoria digital y analizar la incidencia de la tenencia de la tableta del Plan Ibirapitá, de la composición de la red familiar y del tipo de hogar, dejando constantes las otras variables, como control. Esto es posible porque «la ecuación de la función logística permite asignar valores a las variables independientes para generar valores de la dependiente» que permiten predecir su

\footnotetext{
${ }^{5}$ La variable «tenencia de computadora» utilizada incluye laptop, notebook o tabletas en el hogar, excepto tabletas del Plan Ibirapitá.
} 
comportamiento (López-Roldán y Fachelli, 2015, p. 10). Los valores resultantes de dicha ecuación se pueden interpretar «en términos de probabilidad para pronosticar un comportamiento» (López-Roldán y Fachelli, 2015, p. 10). Luego de simular los escenarios, se comprobó si las diferencias entre las probabilidades predichas eran significativas, comparando las esperanzas.

\section{Resultados}

Se corrió el modelo logit en SPSS con 5662 observaciones. El test de Hosmer y Lemeshow resultó no significativo en un valor de $0,51 .^{6}$ Todos los pasos resultaron significativos y el logaritmo de verosimilitud decreció hasta el valor final $(3226,29)$, por lo que el modelo presentado tiene el mejor ajuste predictivo obtenido. En el último paso, el modelo convergió en seis iteraciones y resultó significativo. Este modelo tiene un alto porcentaje de acierto global $(87 \%)$ y de acierto para clasificar los casos de uso $(75 \%)$ y no uso de Internet $(92 \%)$. Las variables resultaron significativas para un $95 \%$ de confianza y explican un $44 \%$ de la varianza ( $\mathrm{R}$ de Cox y Snell $=0,44)$. Es decir, todas las variables incluidas en el modelo producen algún cambio en la probabilidad de uso de Internet entre las beneficiarias del Plan Ibirapitá que tienen 65 años o más (véase Tabla 2).

Siguiendo a Gelman y Hill (2007), se puede aplicar la regla de dividir entre cuatro para analizar el impacto de las variables independientes sobre la dependiente en el centro de los datos, cuando la curva logística alcanza su pendiente máxima. Si se toman los coeficientes de regresión logística (B) y se los divide entre cuatro, se obtiene el impacto máximo que tiene cada una de las variables independientes sobre el uso de Internet. En la tabla 2 se presenta dicho impacto en orden de mayor a menor (B/4): el mayor impacto en la probabilidad de uso de Internet lo genera la tableta Ibirapitá en el hogar $(75 \%)$, seguida de todas las variables de la infraestructura de acceso.

Tabla 2: Estadísticas de la regresión logística

\begin{tabular}{lcccccc}
\hline Variables & B & DS & Wald & Sig. & EXP(B) & B/4 \% \\
\hline $\begin{array}{l}\text { Tableta } \\
\text { Ibirapitá }\end{array}$ & 2,99 & 0,03 & 11396,33 & 0,000 & 19,90 & $75 \%$ \\
$\begin{array}{l}\text { Internet en el } \\
\text { hogar }\end{array}$ & 2,00 & 0,03 & 6386,57 & 0,000 & 7,37 & $50 \%$ \\
$\begin{array}{l}\text { Computadora* } \\
\text { Teléfono }\end{array}$ & 1,71 & 0,03 & 3802,11 & 0,000 & 5,54 & $43 \%$ \\
celular & 1,63 & 0,02 & 4983,27 & 0,000 & 5,08 & $41 \%$ \\
\hline
\end{tabular}

\footnotetext{
${ }^{6}$ Se presenta el test de Hosmer y Lemeshow para evaluar la bondad de ajuste del modelo porque se trabajó con datos no agrupados. Para el test se corrió el modelo no ponderado (Cañadas Reche, 2013).
} 


\begin{tabular}{|c|c|c|c|c|c|c|}
\hline $\begin{array}{l}\text { Liceo o } \\
\text { estudios } \\
\text { superiores } \\
\text { completos** }\end{array}$ & 1,61 & 0,03 & 2849,67 & 0,000 & 5,01 & $40 \%$ \\
\hline Región rural & $-0,83$ & 0,05 & 308,56 & 0,000 & 0,43 & $-21 \%$ \\
\hline $\begin{array}{l}\text { Ingresos per } \\
\text { cápita (log) }\end{array}$ & 0,71 & 0,05 & 228,48 & 0,000 & 2,03 & $18 \%$ \\
\hline $\begin{array}{l}\text { Primaria } \\
\text { completa }\end{array}$ & 0,67 & 0,03 & 710,73 & 0,000 & 1,96 & $17 \%$ \\
\hline $\begin{array}{l}\text { Hijos en el } \\
\text { exterior }\end{array}$ & 0,60 & 0,03 & 547,72 & 0,000 & 1,82 & $15 \%$ \\
\hline $\begin{array}{l}\text { Menores en } \\
\text { hogar }\end{array}$ & $-0,56$ & 0,03 & 275,68 & 0,000 & 0,57 & $-14 \%$ \\
\hline $\begin{array}{l}\text { Hijos en el } \\
\text { hogar }\end{array}$ & $-0,55$ & 0,02 & 540,76 & 0,000 & 0,56 & $-15 \%$ \\
\hline $\begin{array}{l}\text { Hogar } \\
\text { unipersonal }\end{array}$ & 0,31 & 0,02 & 322,23 & 0,000 & 1,71 & $14 \%$ \\
\hline $\begin{array}{l}\text { Pareja en el } \\
\text { hogar }\end{array}$ & 0,31 & 0,02 & 161,85 & 0,000 & 1,36 & $8 \%$ \\
\hline Edad & $-0,08$ & 0,00 & 2992,125 & 0,000 & 0,92 & $-2 \%$ \\
\hline Constante & $-2,72$ & & & 0,000 & & \\
\hline
\end{tabular}

(*) No incluye tableta Ibirapitá.

(**) Categoría de referencia: nivel educativo 1, no tiene estudios o tiene primaria incompleta.

Se trabajó con la muestra ponderada.

El cociente de razones $(\operatorname{EXP}(B))$ indica en cuánto varía la razón entre la probabilidad de uso y no uso de Internet cuando la variable independiente varía en una unidad (véase Tabla 2). Así, quienes viven en hogares donde hay una tableta Ibirapitá tienen 20 veces más chances de usar Internet con respecto a quienes no tienen tableta Ibirapitá; quienes viven en hogares donde hay conexión a Internet tienen siete veces más posibilidades de usar Internet con respecto a quienes no tienen conexión en el hogar. De esa manera, se interpretan todas las variables dicotómicas incluidas en el modelo.

En el caso del nivel educativo, el cambio se debe interpretar en relación con la categoría elegida como referencia. Esto es: quienes han culminado estudios liceales o superiores tienen cinco veces más posibilidades de usar de Internet que quienes no han tenido estudios formales o no han culminado la escuela primaria. Quienes han finalizado los estudios primarios tienen dos veces más posibilidad de usar Internet que quienes no han tenido estudios formales o no han culminado la escuela primaria (véase Tabla 2). 
Cuando se interpretan los valores de las dos variables cuantitativas incluidas en el modelo (ingresos per cápita y edad) se debe tener en cuenta que la tasa de cambio en la posibilidad de uso de Internet alude a la variación por cada unidad de medida de la variable independiente. En el caso de la edad, la tasa de cambio refiere a la diferencia de un año. Entonces, por cada año más que tenga la persona, su posibilidad de usar Internet disminuye 0,92 veces. Si hay una diferencia de diez años entre dos mujeres, la mayor tiene nueve veces menos posibilidades de usar Internet que la más joven (véase Tabla 2).

Dicho en otros términos, se obtuvo un incremento en los odds ratio (OR), cociente de razones o razón de razones, con cada una las variables vinculadas a la infraestructura de acceso: tenencia de tableta Ibirapitá, de conexión a Internet y computadora en el hogar y de teléfono celular. Se confirmó el incremento de OR con el aumento del nivel de ingresos y educativo y su decrecimiento con la vida en zona rural y la edad. En relación con la estructura de la familia y el hogar, se verificó que la presencia de hijos en el exterior aumenta los OR, al igual que vivir sola o tener pareja en el hogar, mientras que la presencia de hijos y de menores en el hogar los disminuye (véase Tabla 2).

Para el análisis con escenarios se creó un perfil de mujer promedio de referencia que tiene 76 años, primaria completa, ingresos mensuales per cápita promedio, no vive sola y en su hogar, urbano, no hay menores, hijos ni pareja; tiene celular, pero no tiene tableta Ibirapitá. La utilidad de construir este perfil promedio es que permite estudiar la probabilidad de uso de Internet de una beneficiaria promedio cuando se modifican otras variables cuya incidencia se quiere indagar. Dicho perfil se comparó en escenarios con divisoria digital y sin divisoria digital. Al evaluar estos diferentes escenarios con el paquete Zelig del software R, se obtuvieron los resultados presentados en las tablas 3 y 4 .

Cuando hay divisoria digital (véase Tabla 3), esta se muestra como la principal barrera para el uso de Internet entre las mujeres mayores en Uruguay, incluso con la puesta en marcha del Plan Ibirapitá. Solo cuando se entrega la tableta Ibirapitá en un hogar con acceso a Internet, la probabilidad de uso de Internet supera el $50 \%$ (es decir, usarán Internet). Si se entrega la tableta Ibirapitá, pero el hogar no tiene Internet o computadora, estas mujeres continuarán sin usar Internet (véase Gráfico 1).

Tabla 3: Probabilidad de uso de Internet en escenarios con divisoria digital (no hay computadora ni Internet en el hogar)

Perfil promedio de referencia: mujer de 76 años, con primaria completa, ingresos per cápita promedio, que vive en zona urbana, en un hogar no unipersonal, sin menores ni hijos o pareja en el hogar y que tiene teléfono celular.

\begin{tabular}{l|c|c}
\hline & \multicolumn{2}{|c}{ Probabilidad de uso de Internet } \\
\hline Perfil promedio de referencia & Sin tableta Ibirapitá & Con tableta Ibirapitá \\
\hline Hay Internet en el hogar & $2 \%$ & $30 \%$ \\
\hline Hay computadora en el hogar & $13 \%$ & $76 \%$ \\
\hline Hay hijos en el exterior & $3 \%$ & $71 \%$ \\
\hline Vive sola & $3 \%$ & $43 \%$ \\
\hline Hay pareja en el hogar & $3 \%$ & $42 \%$ \\
\hline Hay menores en el hogar & $1 \%$ & $37 \%$ \\
\hline
\end{tabular}




\begin{tabular}{l|c|c}
\hline Hay hijos en el hogar & $2 \%$ & $21 \%$ \\
\hline
\end{tabular}

Diferencias significativas con el perfil promedio de referencia

Gráfico 1: Probabilidad de uso de Internet en escenario con divisoria digital (es usuaria de Internet cuando la probabilidad supera el $50 \%$ )

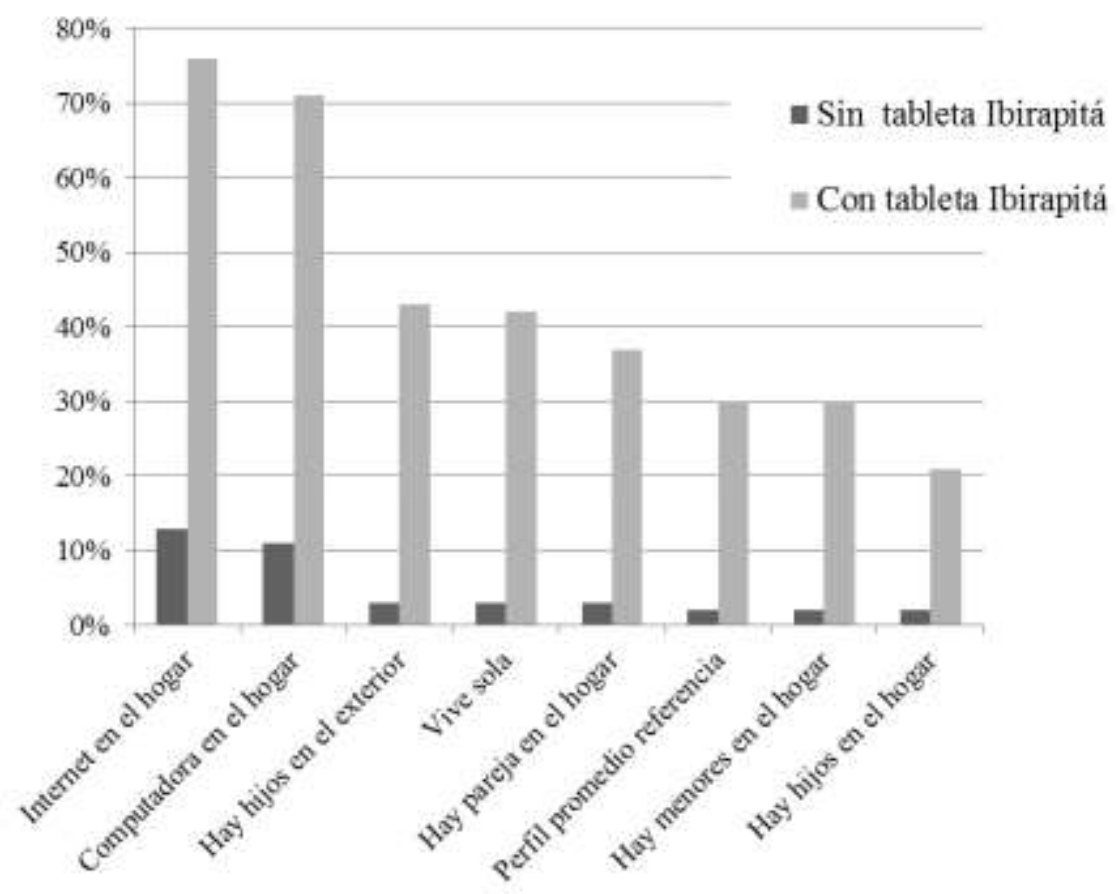

Ahora, ¿qué ocurre en un escenario sin divisoria digital? Para responder a la pregunta 3 se compararon los escenarios en los que el perfil promedio de referencia vive en un hogar con Internet y computadora (que no es del Plan Ibirapitá) con otros escenarios. Los resultados muestran que incluso si hay Internet y computadora en el hogar, pero no hay tableta Ibirapitá, las mujeres no usarán Internet, excepto cuando tengan pareja en el hogar, vivan solas o tengan hijos en el exterior (véase Tabla 4).

Tabla 4: Probabilidad de uso de Internet en escenarios sin divisoria digital (hay computadora e Internet en el hogar)

Perfil promedio de referencia: mujer de 76 años, con primaria completa, ingresos per cápita promedio, que vive en zona urbana, en un hogar sin menores, hijos o pareja y que tiene teléfono celular, además de computadora e Internet. 


\begin{tabular}{r|c|c}
\hline \multirow{2}{*}{} & \multicolumn{2}{|c}{ Probabilidad de uso de Internet } \\
\cline { 2 - 3 } & Sin tableta Ibirapitá & Con tableta Ibirapitá \\
\hline Perfil promedio de referencia & $46 \%$ & $94 \%$ \\
\hline Hay hijos en el exterior & $63 \%$ & $98 \%$ \\
\hline Vive sola & $59 \%$ & $97 \%$ \\
\hline Pareja en el hogar & $55 \%$ & $95 \%$ \\
\hline Hay menores en el hogar & $33 \%$ & $93 \%$ \\
\hline Hay hijos en el hogar & $34 \%$ & $91 \%$ \\
\hline
\end{tabular}

\section{Diferencias significativas con el perfil promedio de referencia}

Los escenarios simulados muestran que, cuando se ve superada la divisoria digital en el hogar - hay computadora e Internet-, la entrega de la tableta Ibirapitá a estas beneficiarias de perfil teórico promedio marca una diferencia significativa en la probabilidad de uso que va del $46 \%$ al $94 \%$. Estas mujeres usarán Internet, aunque no reciban la tableta del Plan Ibirapitá, cuando vivan solas (59 \%), cuando tengan pareja en el hogar (55\%) y cuando tengan hijos en el exterior (63\%). Sin embargo, la brecha gris se mantendrá cuando vivan en hogares con menores de 14 años o con hijos en el hogar (véase Gráfico 2).

Gráfico 2: Probabilidad de uso de Internet en escenario sin divisoria digital (es usuaria de Internet cuando la probabilidad supera el $50 \%$ )

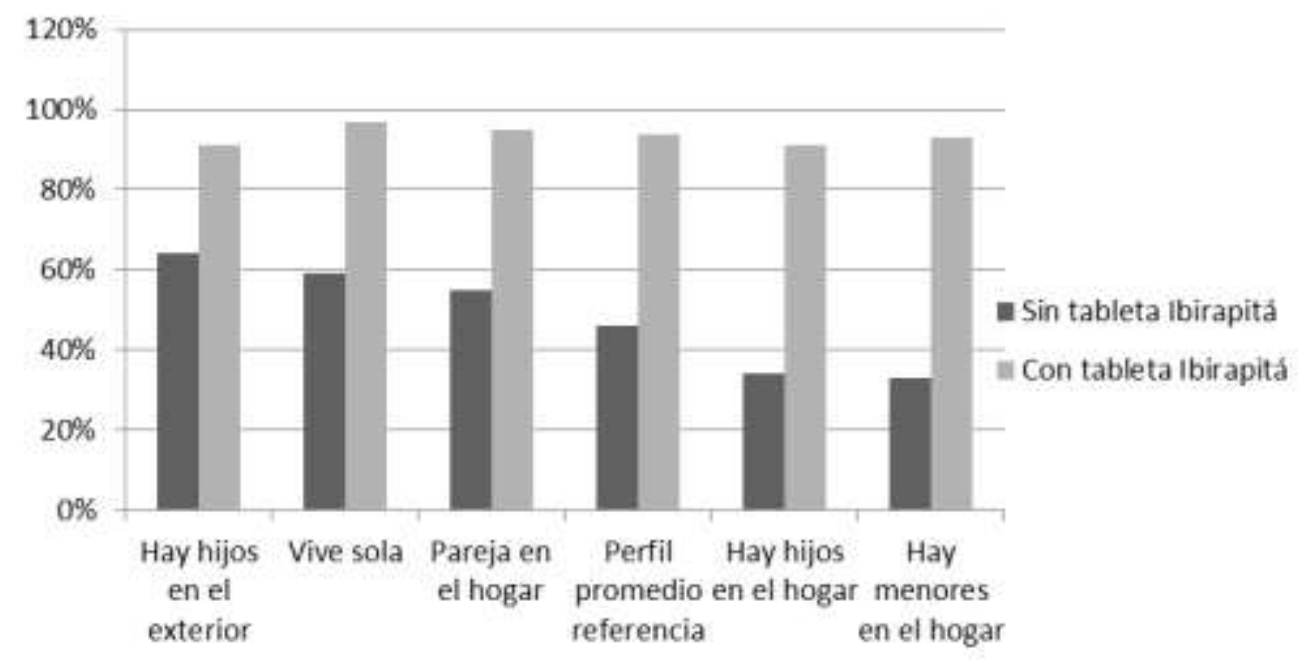

\section{Discusión}

En este modelo, en primer lugar, la falta de infraestructura representa la principal barrera para el uso de Internet entre las mujeres de 65 años o más beneficiarias del Plan Ibirapitá en Uruguay (véase Tabla 2). La infraestructura considerada incluye: tableta Ibirapitá, conexión a Internet en el hogar, computadora en el hogar (que no sea del Plan Ibirapitá) y teléfono celular. De estos cuatro elementos, la tenencia de tableta Ibirapitá y la conexión a Internet son las únicas variables de todo el modelo que tienen un impacto del $50 \%$ o más sobre el uso de Internet. Estos resultados son consistentes con los de Farooq Mubarak y Michael Nycyk (2017), 
quienes indican que la falta de infraestructura es una de las barreras que explican la brecha gris en los países en desarrollo.

El segundo lugar en términos de impacto lo tienen factores socioeconómicos y la distinción entre zona urbana y rural, variables cuya incidencia sobre el uso de Internet es conocida en todas las edades (véase Tabla 2). De estas variables, la única que tiene un impacto negativo es la que distingue al hogar ubicado en una zona rural.

En tercer lugar, incide la composición de la red familiar y la estructura del hogar con un impacto que varía del $8 \%$ al $15 \%$ (véase Tabla 2). En concreto, la presencia de hijos en el exterior actúa como motivador para el uso de Internet y la presencia de hijos en el hogar como freno. Esto remite a las necesidades instrumentales referidas por Ferreira, Sayago y Blat (2016), dado que la necesidad de comunicarse con un hijo que está lejos motiva a estas mujeres para usar Internet. Además, subraya la necesidad de considerar la posibilidad de que las relaciones intergeneracionales impacten de forma negativa; no solo por la presencia de hijos en el hogar, sino porque en este modelo la presencia de menores de 14 años en el hogar también impacta negativamente sobre la probabilidad de uso de Internet entre las beneficiarias. Estos resultados son consistentes con la ambigüedad vinculada a la influencia de las relaciones intergeneracionales sobre el uso de Internet. A menudo se asume —en particular desde las políticas públicas- que los vínculos intergeneracionales resultan un estímulo exclusivamente positivo para la adopción y el uso de tecnologías digitales entre las personas mayores. Los resultados de este trabajo contradicen dicha asunción en relación con el uso de Internet entre mujeres mayores. Como hipótesis se puede proponer que, cuando una mujer mayor vive con hijos o nietos en el hogar, son ellos quienes utilizan los dispositivos de acceso a Internet que hay en él. Si bien hay computadora en el hogar, la mujer mayor no tiene acceso a ella. Los hijos y los nietos conviviendo en el hogar se presentarían, así, como una barrera para el uso de Internet en el entorno del hogar (véase Tabla 2).

Por último, el menor valor del impacto máximo de todas las variables es negativo y corresponde a la edad. Aunque se presenta en la tabla 2 como el menor impacto individual de todas las variables cuando aumenta en un año la edad de la mujer, resulta más adecuado evaluar su impacto en intervalos. En concreto, cada diez años la posibilidad de usar Internet disminuye nueve veces. Una mujer de 75 años tendría nueve veces menos chances de usar Internet que una mujer de 65 años. Interpretada de esta forma, después de la tenencia de la tableta Ibirapitá, la edad sería la variable que más incidencia tiene en la posibilidad de uso de Internet (véase $\operatorname{EXP}(B)$ en Tabla 2). Además de la edad, entre las variables que podemos interpretar como indicadoras de las modificaciones relacionadas con el curso de la vida se encuentra el hogar unipersonal, ya que el hecho de vivir sola, entre las mujeres de 65 años o más, corresponde, en la mayoría de los casos, a los cambios en el tipo de hogar que se siguen de la pérdida del cónyuge. Las mujeres que viven en hogares unipersonales, de acuerdo a los resultados de este modelo, tienen dos veces más posibilidades de usar Internet con respecto a las que no viven en hogares unipersonales (véase Tabla 2). Otro aspecto que refiere a los cambios en el curso de la vida es el momento del retiro de la vida laboral, el cual está 
considerado implícitamente en este modelo, ya que todas las beneficiarias son jubiladas. Por otro lado, dado que con la edad cambia la estructura y composición del hogar, las variables de la estructura del hogar y familiar podrían variar según el tramo de edad considerado dentro del grupo de 65 años o más. Como línea de trabajo se podría realizar un análisis con este modelo en diferentes tramos de edad, siempre que se cuente con la cantidad suficiente de observaciones por grupo, para seguir trabajando con la edad como variable cuantitativa incluida en él. $^{7}$

A partir de este modelo, se compararon escenarios para evaluar la incidencia de la entrega de la tableta del Plan Ibirapitá en condiciones de divisoria digital (que son las actuales) y sin divisoria digital (que son las ideales en cuanto a infraestructura) a una beneficiaria de perfil promedio. Por un lado, cuando el Plan Ibirapitá ha llegado a todas las beneficiarias de 65 años o más con este perfil, en condiciones de divisoria digital — configurada por la ausencia de conexión a Internet y de computadora en el hogar- es probable que aquellas que no tengan Internet en el hogar no usen Internet (véase Tabla 3). Las mujeres beneficiarias del Plan Ibirapitá con 65 años o más que no tenían Internet en el hogar representaban el $48 \%$ de las beneficiarias de este grupo de edades en 2016. Mientras en Montevideo el porcentaje era menor (39\%), fuera de la capital el porcentaje de beneficiarias que vivían en hogares sin conexión a Internet era aún mayor (55\%) (consulta propia a ECH, INE, 2016).

Por otro lado, en condiciones sin divisoria digital - es decir, hay Internet y computadora en el hogar, tienen teléfono celular, pero no recibieron la tableta Ibirapitá-, es probable que las mujeres de 65 años o más con este perfil de beneficiarias no usen Internet, salvo que tengan hijos en el exterior, vivan solas o en pareja (véase Tabla 4). Las beneficiarias de este perfil que vivan con menores o hijos en el hogar no usarán Internet, a pesar de tener computadora y conexión a Internet en el hogar. Este resultado sostiene la pertinencia de la entrega de la tableta, incluso a mujeres que tienen Internet y computadora en el hogar, al menos en estos casos. Puede sugerirse como hipótesis que la tenencia de un dispositivo personal y no del hogar es relevante para el uso de Internet; mientras que la computadora que hay el hogar no les pertenece necesariamente - excepto que vivan solas-, la tableta que les brinda el Plan Ibirapitá es suya.

En un escenario ideal, sin divisoria digital - hogares con acceso a Internet y computadora - la brecha gris se superaría con el Plan Ibirapitá en la zona urbana considerando el perfil promedio (véase Tabla 4).

Es importante observar que este modelo explica mejor el no uso de Internet que su uso entre las beneficiarias del Plan Ibirapitá con 65 años o más, a pesar de que el porcentaje de acierto es alto en ambos casos. El porcentaje de acierto para los casos de uso de Internet es de $75 \%$. $^{8}$

\footnotetext{
${ }^{7}$ Es preferible aplicar el modelo por tramos de edad que categorizar una variable cuantitativa para incluirla en el modelo logístico.

${ }^{8} \mathrm{La}$ ECH solo recopila datos sobre los hijos de las mujeres de 14 años o más. Esto impide correr este modelo en la población masculina.
} 
En definitiva, se presentan cuatro aspectos que inciden en la brecha gris en Uruguay para el perfil de mujeres beneficiarias del Plan Ibirapitá estudiado. El primero es la infraestructura de acceso a Internet, que representa la principal barrera para superar la brecha gris. El segundo aspecto lo conforman las variables socioeconómicas (ingresos, nivel educativo) y la distinción entre zona urbana y rural. Hasta aquí se trata de factores que de una forma u otra se pueden alcanzar desde las políticas públicas. El tercer aspecto tiene que ver con la estructura y la composición del hogar donde hay estímulos y barreras. Estos aspectos de la composición del hogar impactan sobre el uso de Internet incluso cuando se ha superado la brecha de infraestructura de acceso a Internet y los hogares tienen computadora y conexión a la red. Por último, si bien cuando se van sumando los años la posibilidad de usar Internet disminuye, la entrega de la tableta Ibirapitá puede ayudar a compensar esa variación en la tasa de uso de Internet relacionada con el paso del tiempo.

\section{Consideraciones finales}

El aporte principal de este trabajo es el modelo logístico como herramienta que permite predecir el impacto del Plan Ibirapitá en diferentes escenarios. Este modelo es una respuesta - con las restricciones propias del trabajo con datos secundarios - a la pregunta sobre cuáles son los factores que inciden en el uso de Internet entre las mujeres mayores beneficiarias del Plan Ibirapitá en Uruguay: la infraestructura tecnológica, la estructura del hogar y de la red familiar, y los más conocidos factores socioeconómicos. Los resultados presentados están sujetos al error y a las limitaciones de trabajar con datos que no fueron recopilados con el objetivo de realizar este análisis. El estudio de datos recogidos con este fin permitirá confirmar o rebatir estos resultados para ajustar el modelo. Es importante tener en cuenta que este análisis se realizó entre las mujeres de 65 años o más con perfil de beneficiarias del Plan Ibirapitá para analizar el impacto que tiene la puesta en marcha de esta política pública sobre la población a la que apunta. Aunque resulte evidente, es necesario explicitar que dicho perfil no alcanza a todas las mujeres de 65 años o más del Uruguay ni alcanza a las de menores recursos económicos. La brecha gris en Uruguay se extiende más allá del perfil de las beneficiarias del Plan Ibirapitá. En futuras líneas de trabajo se abordará el análisis en toda la población de mujeres de 65 años o más.

El trabajo con escenarios promedio tiene la ventaja de permitir controlar las variables socioeconómicas en valores promedio, para analizar el impacto de las otras variables incluidas en el modelo, en el supuesto de que el promedio dice algo en común acerca de la población en estudio. En un contexto de divisoria digital, como el de Uruguay, la entrega de la tableta no es un estímulo suficiente para alentar el uso de Internet entre las beneficiarias del Plan Ibirapitá con el perfil promedio analizado. Se requiere entregar la tableta Ibirapitá junto a la conexión a Internet en el hogar para lograr un impacto sobre el uso de Internet y reducir la brecha gris.

Cuando se ha superado la divisoria digital, el Plan Ibirapitá sigue siendo importante para superar la brecha gris en el grupo de mujeres con perfil promedio que viven en hogares con menores de 14 años o con hijos en el hogar. Qué tanto el 
impacto de la entrega de la tableta Ibirapitá deriva de la necesidad de un dispositivo propio o de las características de la tableta como dispositivo de acceso móvil a Internet es una línea a estudiar. En los casos de mujeres de perfil promedio que viven solas, en pareja o que tienen hijos en el exterior no es necesario el Plan Ibirapitá para que utilicen Internet cuando ya tienen computadora y acceso a Internet en el hogar.

Por último, al trabajar con valores promedio se deja afuera la variabilidad que podría encontrarse en diferentes estratos socioeconómicos y edades. Es posible que la estructura del hogar incida de manera diferente de acuerdo a la edad y el estrato socioeconómico. Esta es otra línea para indagar en futuros trabajos.

\section{Agradecimientos}

Agradezco a la Dra. Andrea Quadrelli y la Dra. Mireia Fernández-Ardèvol la sugerencia de algunas de las referencias bibliográficas.

\section{Referencias bibliográficas}

ALDANA, G.; GARCÍA, L.; JACOBO, A. (2012). Las Tecnologías de la Información y Comunicación (TIC) como alternativa para la estimulación de los procesos cognitivos en la vejez. CPU-e, Revista de Investigación Educativa, 14. Recuperado de http://www.uv.mx/cpue/num14/practica/aldana_garcia_mata_tic_vejez.html

ANTONUCCI, T. C.; AJROUCH, K. J.; BIRDITT, K. S. (2014). The convoy model: Explaining social relations from a multidisciplinary perspective. The Gerontologist, 54(1), 82-92. doi: 10.1093/geront/gnt118

ARBER, S.; DAVIDSON, K.; GINN, J. (2003). Gender and ageing. Maidenhead-Filadelfia: Open University Press.

BANDURA, A. (1977). Social learning theory. Englewood Cliffs: Prentice-Hall.

BANDURA, A. (1994). Social cognitive theory of mass communication. En J. Bryant y D. Zillman (Eds.), Media effects: Advances in theory and research (pp. 61-89). Hillsdale: Lawrence Erlbaum.

BARNARD, Y. et al. (2013). Learning to use new technologies by older adults: Perceived difficulties, experimentation behaviour and usability. Computers in Human Behavior, 29(4), 1715-1724. doi: 10.1016/j.chb.2013.02.006

BARRANTES CÁCERES, R.; COZZUBO CHAPARRO, A. (2017). Age for learning, age for teaching: The role of inter-generational, intra-household learning in Internet use by older adults in Latin America. Information, Communication \& Society, 1-17. doi:10.1080/1369118X.2017.1371785

BINOTTI, P.; SPINA, D. (2009). Funciones ejecutivas y aprendizaje en el envejecimiento normal: Estimulación cognitiva desde una mirada psicopedagógica. Revista Chilena de Neuropsicología, 4(2), 119-126.

BLASCHKE, C. M.; FREDDOLINO, P. P.; MULLEN, E. E. (2009). Ageing and technology: A review of the research literature. British Journal of Social Work, 39(4), 641-656. doi: 10.1093/bjsw/bcp025 
BOGLIACCINI, J. A.; RODRÍGUEZ, F. (2015). Regulación del sistema educativo y desigualdades de aprendizaje en el Uruguay. Revista CEPAL. Recuperado de http://200.9.3.98/handle/11362/38798

BROADY, T.; CHAN, A.; CAPUTI, P. (2010). Comparison of older and younger adults' attitudes towards and abilities with computers: Implications for training and learning. British Journal of Educational Technology, 41(3), 473-485.

BROWN, S. A.; VENKATESH, V.; BALA, H. (2006). Household technology use: Integrating household life cycle and the model of adoption of technology in households. The Information Society, 22(4), 205-218. doi:10.1080/01972240600791333

CABALLERO DE LUIS, S. (2014). Internet, vejez y redes sociales en Uruguay: ¿se relacionan el soporte social e Internet? Revista de Ciencias Sociales, DS-FCS, 27(35), 137-160.

CABALLERO DE LUIS, S. (2016). Internet, familia y hogares entre las mujeres mayores en Uruguay. Universitat Oberta de Catalunya. Recuperado de https://www.researchgate.net/publication/311453428_Internet_familia_y_hogares _entre_las_mujeres_mayores_en_Uruguay

CAÑADAS RECHE, J. L. (2013). Regresión logística. Tratamiento computacional con $R$. (Tesis de maestría en Estadística Aplicada, Universidad de Granada). Recuperado de https://www.researchgate.net/profile/Jose_CanadasReche/

CHAFFIN, A. J.; HARLOW, S. D. (2005). Cognitive learning applied to older adult learners and technology. Educational Gerontology, 31(4), 301-329. doi:10.1080/03601270590916803

CHANG, J.; MCALLISTER, C.; MCCASLIN, R. (2015). Correlates of, and barriers to, Internet use among older adults. Journal of Gerontological Social Work, 58(1), 66-85. doi:10.1080/01634372.2014.913754

CHOI, N. G.; DINITTO, D. M. (2013). The digital divide among low-income homebound older adults: Internet use patterns, eHealth literacy, and attitudes toward computer/Internet use. Journal of Medical Internet Research, 15(5), 93. doi:10.2196/jmir.2645

CORREA, T. et al. (2013). Brokering new technologies: The role of children in their parents' usage of the Internet. New Media \& Society.

CUTLER, S.; HENDRICKS, J.; GUYER, A. (2003). Age differences in home computer availability and use. The Journals of Gerontology, 58(B), 271-280.

CZAJA, S. J.; SHARIT, J. (2012) Designing training and instructional programs for older adults. Boca Ratón: CRC Press.

DAMODARAN, L.; SANDHU, J. (2016). The role of a social context for ICT learning and support in reducing digital inequalities for older ICT users. International Journal of Learning Technology, 11(2), 156-175.

DANNEFER, D. (1987). Aging as intracohort differentiation: Accentuation, the Matthew effect, and the life course. Sociological Forum, 2, 211-236.

DANNEFER, D.; SETTERSTEN, R. A. (2010). The study of the life course: Implications for social gerontology. En The SAGE handbook of social 
gerontology (pp. 4-20). Sage.

DE BENITO-CASTANEDO, J. (2017). Análisis bibliográfico sobre la brecha digital y la alfabetización en nuevas tecnologías. Revista Electrónica Educare, 21(2), 1. doi: 10.15359/ree.21-2.9

FERREIRA, S. M.; SAYAGO, S.; BLAT, J. (2016). Going beyond telecenters to foster the digital inclusion of older people in Brazil: Lessons learned from a rapid ethnographical study. Information Technology for Development, 22(1), 26-46. doi 10.1080/02681102.2015.1091974

FRIEMEL, T. N. (2016). The digital divide has grown old: Determinants of a digital divide among seniors. New Media \& Society, 18(2), 313-331.

GALPERIN, H. (2017). Why are half of latin americans not online? A four-country study of reasons for Internet non-adoption. International Journal of Communication, 11, 3332-3354.

GELMAN, A.; HILL, J. (2007). Data analysis using regression and multilevel/hierarchical models. Nueva York: Cambridge University Press.

HAEDERLE, M. (2011). Technology fear stops older adults from logging on: But scientists are breaking the computer block. AARP Bulletin. Recuperado de http://www.aarp.org/technology/innovations/info-08-2011/elderly-fear-oftechnology.3.html

HAIR, J. et al. (2010). Multivariate data analysis (7. ${ }^{\text {e ed.). }}$

HARGITTAI, E.; DOBRANSKY, K. (2017). Old dogs, new clicks: Digital inequality in skills and uses among older adults. Canadian Journal of Communication, 42(2), 195-212. doi:10.22230/cjc2017v42n2a3176

HELSPER, E. (2009). The ageing Internet: Digital choice and exclusion among the elderly. Working with Older People, 13(4), 28-33.

HELSPER, E. (2010). Gendered Internet use across generations and life stages. Communication Research, 37(3), 352-374. doi: 10.1177/0093650209356439

Instituto Nacional de Estadística, Uruguay (2016). Encuesta continua de hogares 2016. Recuperado de http://www.ine.gub.uy/web/guest/encuesta-continua-dehogares1

LÓPEZ-ROLDÁN, P.; FACHELLI, S. (2015). Análisis de regresión logística. En Metodología de la Investigación Social Cuantitativa, 1. a ed., (pp. 5-56). Bellaterra (Cerdanyola del Vallès): Dipòsit Digital de Documents, Universitat Autònoma de Barcelona. Recuperado de http://ddd.uab.cat/record/163570

MA, Q. et al. (2015). Acceptance of ICTs by older adults: A review of recent studies. En J. Zhou y G. Salvendy (Eds.), Human aspects of IT for the aged population. Design for aging, Vol. 9193 (p. 239). Los Ángeles: Springer.

MERRIAM, S. B.; CAFFARELLA, R. S.; BAUMGARTNER, L. M. (2012). Learning in adulthood: A comprehensive guide. John Wiley \& Sons.

MILLWARD, P. (2003). The "grey digital divide": Perception, exclusion and barriers of access to the Internet for older people. First Monday, 8(7). doi:.5210/fm.v8i7.1066.

MORI, K.; HARADA, E. T. (2010). Is learning a family matter?: Experimental 
study of the influence of social environment on learning by older adults in the use of mobile phones: Is learning a family matter? Japanese Psychological Research, 52(3), 244-255. doi: 10.1111/j.1468-5884.2010.00434.x

MUBARAK, F.; NYCYK, M. (2017). Teaching older people Internet skills to minimize grey digital divides: Developed and developing countries in focus. Journal of Information, Communication and Ethics in Society, 15(2), 165-178. doi: 10.1108/JICES-06-2016-0022

PETRETTO, D. R. et al. (2016). Envejecimiento activo y de éxito o saludable: Una breve historia de modelos conceptuales. Revista Española de Geriatría y Gerontología, 51(4), 229-241. doi:10.1016/j.regg.2015.10.003

PHILliPS, J.; AJROUCH, K.; HILLCOAT-NALLÉTAMBY, S. (2010). Key concepts in social gerontology. Sage.

Plan Ibirapitá. Plan de inclusión digital de jubilados. (s. f.). Recuperado de http://ibirapita.org.uy/

SCHEERDER, A.; VAN DEURSEN, A.; VAN DIJK, J. (2017). Determinants of Internet skills, uses and outcomes: A systematic review of the second -and thirdlevel digital divide. Telematics and Informatics. doi: 10.1016/j.tele.2017.07.007

SILVA, E. B. (2010). Technology, culture, family: Influences on home life. Palgrave Macmillan, UK.

TSAI, H. S.; SHILLAIR, R.; COTTEN, S. R. (2015). Social support and "playing around": An examination of how older adults acquire digital literacy with tablet computers. Journal of Applied Gerontology. 36(1), 29-55. doi:10.1177/0733464815609440

VAN DEURSEN, A., HELSPER, E. J., EYNON, R., y VAN DIJK, J. A. G. M. (2017). The compoundness and sequentiality of digital inequality. International Journal of Communication, 11, 452-473.

World Health Organisation. (2002). Active ageing: A policy framework.

Nota: Soledad Caballero es Magister en Investigación en Sociología por la Universidad de Barcelona; candidata a doctora por la Universitat Oberta de Catalunya. 